\title{
The Effect of Isokinetic Testing Speed on the Reliability of Muscle Fatigue Indicators During a Hip Fatigue Protocol
}

\begin{abstract}
The aim of this study was to investigate the reliability of fatigue indicators calculated from peak torque and total work during isokinetic speeds of $60,90,120$ and $180^{\circ} \cdot \mathrm{s}^{-1}$ during a hip fatigue protocol. Ten males suffering from a history of unilateral functional ankle instability and ten male healthy controls performed five maximal concentric contractions on an isokinetic dynamometer. Following a four minute rest period subjects were instructed to perform repeated maximal concentric contractions to fatigue, which was defined as three consecutive repetitions below $50 \%$ of the maximum peak torque value. Each testing speed was randomised with 24 hours between speeds. The subjects were asked to return to the laboratory seven days later to repeat the four speeds, with 24 hours between speeds. Muscle fatigue was determined for each testing speed by the fatigue index, the percent decrease in performance and the slope of the regression equation. The most reliable fatigue determination method was the slope of the regression equation,

when testing at a speed of $120^{\circ} \cdot \mathrm{s}^{-1}$. It is recommended that future investigators examine and plot their data before choosing the slope of the regression equation as their fatigue indicator, as a linear model is required.
\end{abstract}

\section{Key words}


Intraclass correlation coefficient; Standard error of measurement; Functional ankle instability; Fatigue index; Percent decrease in performance; Slope of the regression equation.

\section{Introduction}

Hislop and Perrine [2320] originally introduced the concept of isokinetic dynamometry in 1967. Since then it has become a popular method for the assessment of muscle performance, using common parameters such as peak torque and total work [1916].

Recently, isokinetic dynamometry has been the favoured choice for fatigue assessment $[3, \underline{7} 5]$.

Fatigue has been defined as any reduction in the force generating capacity of the total neuromuscular system regardless of the force required in any given situation [2]. The ability to objectively document muscle fatigue has been an area of concern in both research and clinical settings [2017]. Researchers and healthcare professionals should adopt a reliable method to quantify this manifestation of exercise. The development of isokinetic dynamometry has provided a stepping stone towards objectively measuring muscle fatigue. It should be kept in mind that the reliability of isokinetic testing for a desired protocol should be sufficient so that measures for training or injury induced changes in muscle ability are not attributed to instrument or testing error.

It has been consistently demonstrated that isokinetic peak torque and total work are reliable measures $[3, \underline{4,21,39,43} 18,33,37]$. In regards to the assessment of muscle 
fatigue using this modality, research is sparse and questionable reliability values have been demonstrated $[19,30,3916,26,33]$. Furthermore, the vast majority of studies using isokinetic methods have focused on peak torque, rather than total work [2017]. Peak torque represents only one point of the moment-angular position curve, the highest one [3]. It may not adequately describe other torques developed throughout the movement. This is the reason why total work, which represents the area under the curve [2320], should also be considered. This parameter accounts for the overall adaptation of the curve, not only its highest value.

There is also a lack of consensus in the literature regarding the techniques used to calculate fatigue. The original recommendations proposed by Thorstensson and Karlsson [4638] stated that muscle fatigue should be measured with the fatigue index (FI), calculated as the ratio of the average peak torque of the last three contractions to the average peak torque of the first three contractions [3]. More recently, Pincivero et al. [3933] acknowledged that given the linear nature of the relationship between the total work of each contraction and the number of maximal concentric contractions, the slope of the regression equation would be more appropriate to determine the rate of decrease of total work and thus to estimate muscle fatigue. Although not specific to isokinetic dynamometry, Glaister et al. [1815] quantified muscle fatigue during repeated maximal sprints using the percent decrement score; they argued the suitability of this method since it considered data from each effort in its calculation.

To date, the knee has been the focus for the majority of the isokinetic dynamometry

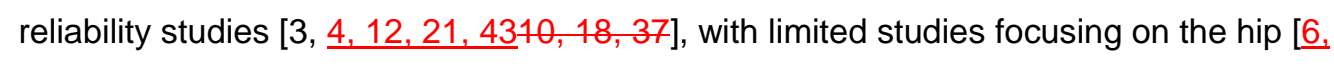


$\underline{404}, 34]$. In addition to this there is negligible research investigating the reliability of different muscle fatigue indicators [3]. At present, there has been no research on the development of a reliable fatigue protocol of the hip musculature.

The validity of isokinetic dynamometry has repeatedly been demonstrated $[10,25,27,37$, 51]. Taylor et al. [45] demonstrated the mechanical validity of the Biodex isokinetic dynamometer in relation to human torque, joint position and limb velocity. More specifically, the validity of a hip abduction protocol $(r=0.65)$ has previously been shown by Katoh et al. [29].

Reliability studies are frequently performed on healthy populations [3, 4, 43, 45], however, isokinetic dynamometry is commonly used to test subjects that are recovering from injury. It is important that equipment is shown to be reliable in healthy populations, but also that it is reliable in persons that have recovered from injury. The lateral ligament complex is the most frequently injured structure in the ankle joint [50], representing $45 \%$ [30] to $95 \%$ of all ankle sprains [35]. The incidence of functional ankle instability (FAI) exhibiting residual symptoms such as feelings of instability, giving way, pain or re-injury, has varied from $10 \%$ [14] up to $80 \%$ in ankle sprain sufferers [44]. Many sufferers of FAl go months or years without suffering an ankle sprain, and are therefore termed healthy patients but with a history of FAl. Clinicians and health professionals in sport will often use isokinetic dynamometry to test this population, as well as healthy individuals, throughout the sporting season. It is therefore important that the equipment used is reliable in both healthy subjects, and patients with a history of FAl. 
It has been suggested that the hip abductors play a critical role in controlling foot placement during ambulation. A deficit at the hip abductors may alter foot placement, causing the foot to contact the ground in a more adducted position [1512]. In patients with FAI functional ankle instability (FAl) this potentially increases the chance of rolling over on the ankle and sustaining a lateral ankle sprain. In addition to this, the added factor of localised hip muscle fatigue in FAI sufferers may further increase the probability of suffering an ankle sprain [1512].

Therefore, the purpose of this study was to determine the effect of isokinetic testing speed on the relative and absolute reliability of the fatigue index, percent decrease in performance and slope of the regression equation during a hip fatiguing protocol, in subjects with a history of FAlpatients and healthy controls.

\section{Method}

\section{Subjects}

\section{A convenience sample was used to recruit ten male subjects with a history of unilateral} functional ankle instability Ten male subjects who suffered from unilateral functional ankle instability $($ age $=($ Mean $\pm S D) 21.05 \pm 4.35$ years, height $=178.95 \pm 5.50 \mathrm{~cm}$, and mass $=$ $78.65 \pm 6.63 \mathrm{~kg})$ and ten male healthy controls (age $=20.5 \pm 3.35$ years, height $=180.45$ $\pm 5.71 \mathrm{~cm}$, and mass $=79.25 \pm 6.21 \mathrm{~kg}$ ) were recruited for this study. Nineteen subjects with a history of FAl volunteered for the study, of which ten matched the inclusion and 


\section{exclusion criteria. Ten healthy subjects were then matched for age, weight and height, out of seventeen healthy volunteers.}

Functional ankle instability has been defined as the subjective feeling of ankle instability or recurrent, symptomatic ankle sprains (or both) due to the proprioceptive and neuromuscular deficits [1512]. However, the subjects with a history of FAI in the present study had not suffered an ankle sprain for a minimum of 6 months, so were currently deemed healthy but with a history of FAI. Institutional ethical approval was granted for this study, and all subjects provided written informed consent before participation. This study was conducted in accordance with recognised ethical standards and national/international laws [2219]. Inclusion criteria included males, aged 18-25 years, who participated in semiprofessional football (two training sessions and one match per week) and who were right leg dominant. The dominant leg was defined as the preferred kicking leg and in the unilateral FAl group the right ankle was the injured ankle.

Subjects were excluded from the study if they had a cold, flu, inner ear or sinus infection in the last two weeks, if they suffered from any musculo-skeletal injuries, knee or hip injuries, fractures to the lower limbs in the last year, or if they suffered from visual impairments, vestibular deficits, or signs of injury such as pain or swelling in their ankles. The subjects with a history of FAI-subjects were required to fill out the FAI questionnaire. Developed by Hubbard and Kaminiski [26르] this validated questionnaire required subjects to answer "yes" to questions 3, 5, 6, 7 and 9, and "no" to questions 4, 8 and 10. Following satisfactory completion of the questionnaire, both of the subject's ankles were examined by the investigator to rule out mechanical instability via the anterior drawer and 
talar tilt test. These tests have been proven as valid [1, $\underline{9} 7]$, however, Bahr et al. [1] suggested that the sensitivity of these tests were improved when the anterior drawer test was performed with the ankle in a plantar-flexed position, and the talar tilt test was performed with the ankle in a dorsi-flexed position. The subject's opposite uninjured ankle acted as the control.

\section{Experimental Design}

Subject's age, mass and height was recorded. Testing was performed on the Biodex System 2 Isokinetic Dynamometer (Biodex Medical Systems, Shirley, New York). The system reliability of the Biodex dynamometer has been shown to be high, with Intraclass Correlation Coefficient's $(\operatorname{ICC}(2,1))$ ranging from $0.92-0.98$ for peak torque, 0.88 to 0.97 for total work and $\underline{0.86}$ to 0.97 for average power [4]. with an Intraclass Correlation Coefficient $($ ICG $(2,1)$ ) of 0.99 for position, peak torque and velocity [8]. The Biodex was set up according to the Biodex System 2 Manual $_{2}$ and was calibrated according to manufacturer's specifications prior to testing. The cushion control was set to zero, to allow the subject the greatest availability of velocity attainment prior to deceleration [5]. All subjects completed a practice session on the isokinetic dynamometer a week prior to the main testing procedure.

Subjects were required to lie on their side (facing away from the dynamometer power head) with the tested hip (right) superior to the opposite hip. The knee of the tested leg was extended, and the opposite knee was flexed at $90^{\circ}$. The axis of the dynamometer was aligned superior and medial to the greater trochanter of the tested leg. The subject's right leg was attached to the Biodex hip attachment, superior to the lateral knee joint line. The 
subject's range of motion was set between $0-45^{\circ}$ of abduction. The degrees of motion were set based on the average limitations of hip motion in healthy individuals $[11,33,419,28$, 35]. The torso and pelvis were stabilised using the straps of the dynamometer and the subject's top hand grasped the border of the chair. The side lying single chair hip abduction/adduction protocol was chosen, as opposed to a standing hip abduction/adduction protocol, due to the chair and straps of the isokinetic dynamometer being able to control for torso and pelvis movement. It may be argued that standing hip abduction/adduction has more relevance to walking, however, the design of the system 2 dynamometer does not allow for support of the torso and pelvis during the standing protocol. Weir et al. [47] found a decrease in peak torque when extraneous artifacts were not controlled for. The authors stated that strict stabilization of the torso was necessary to eliminate these extraneous artifacts, and allow the subject to develop maximum peak torque values. Therefore, the side lying abduction/adduction protocol was deemed more appropriate in the present study, as any unnecessary torso or pelvis movement could be limited.

The subject was then instructed to perform five isokinetic maximal repetitions, to determine their maximum peak torque. Each subject was instructed to push their leg upwards (abduction) and pull their leg downwards (adduction) as hard and as fast as possible. The maximum peak torque value was established and subjects were given a four minute rest period [4236]. Following this, subjects were instructed to abduct and adduct their hip repeatedly as hard and as fast as possible until they reached fatigue. Fatigue was defined as three consecutive repetitions below $50 \%$ of the maximum peak torque value $[11,16,429$, 13]. The same strong verbal encouragements were given to each subject throughout the test to motivate them to develop maximal torque during each repetition [3429]. Each testing 
speed $\left(60,90,120\right.$ and $\left.180^{\circ} \cdot \mathrm{s}^{-1}\right)$ was randomised with a minimum of 24 hours between speeds. The subjects were then given a seven day rest period, and were asked to return to the laboratory to repeat the four speeds again with a minimum of 24 hours between speeds. Therefore, each subject visited the laboratory on eight separate occasions to complete all testing sessions. Subjects were asked to refrain from any vigorous exercise during the week, and were tested at the same time of day to reduce the effect of diurnal variation.

The validity of isokinetic dynamometry has repeatedly been demonstrated $[21,23,31,40]$. Brouin et al. [8] also demonstrated the mechanical validity of the Biodex isokinetic dynamometer in relation to torque, position and velocity $\left(<300^{\circ} \cdot s^{-1}\right)$. More specifically, the validity of a hip abduction protocol has previously been shown by Katoh et al. [25] ( $r=0.65)$. This paper aims to exclusively assess absolute and relative reliability.

\section{Data Analysis}

It has previously been shown that there is an inverse relationship between load range and velocity during concentric contractions [5]. Brown et al. [5] stated if the pre-set velocity is not reached the result is an absence of machine offered resistance. All velocities $(60,90,120$ and $180^{\circ} \cdot \mathrm{s}^{-1}$ ) were reached by all subjects in the present study, but load range did show an inverse relationship to velocity (figure 1), as previously stated by Brown et al. [5]. Therefore, prior to analysis of the peak torque and total work data, all data was reduced for load range.

Peak torque $(\mathrm{N} \cdot \mathrm{m})$ and total work $(\mathrm{J})$ were calculated for each repetition, following load range reduction, and totalled to compute cumulated performance. Muscle fatigue was only 
determined for the hip abductors, as the gluteus medius was the focused muscle for the fatigue protocol, due to its stabilising role in the frontal plane at the hip. Hip abductor muscle fatigue was determined when the procedure was performed at $60,90,120$ and $180^{\circ} \cdot \mathrm{s}^{-1}$.

Fatigue was calculated by the following three methods: the fatigue index [2824], the slope of the regression equation [3933] and the percent decrease in performance [1815]. The fatigue index was calculated by the following equation:

$\mathrm{FI}=100-(($ Average performance of last 3 reps/Average performance of first 3 reps $) \times 100)$

Where performance represented peak torque or total work. The slope was determined via linear regression by plotting performance (i.e. peak torque or total work) against each repetition, for each subject. The slope of the regression line was added to the graph, and using the mathematical formula $y=m x+c$, the $m$ value was taken as the slope, which represented the rate of decrease in performance during the test. The percent decrease in performance was calculated by the following equation:

$\mathrm{DP}=100-([$-Cumulated performance/(Maximal performance $\times n)]+\times 100)$

Where performance represented peak torque or total work, maximal performance represented PTmax or TWmax and $\mathrm{n}$ was the number of repetitions. 


\section{Statistical Analysis}

Using SPSS (Version 19.0) normal Gaussian distribution of the data was verified by the Shapiro-Wilk test. Systematic bias, which refers to a difference in measurements in a particular direction between repeated tests, was assessed with seven [peak torque ( 3 fatigue indicators) and total work ( 3 fatigue indicators) and number of repetitions to fatigue] threeway [group (healthy or healthy with a history of $\mathrm{FAI}) \times \operatorname{speed}\left(60,90,120\right.$ and $\left.180^{\circ} \cdot \mathrm{s}^{-1}\right) \times$ time (first week testing or second week testing)] mixed factorial analysis of variance (ANOVA) $(p<0.05)$. The two within-subject factors were speed and time of test, and the between-subject factor was group type. Sphericity was verified for all data being compared by the Mauchley test. Systematic bias, which refers to a difference in measurements in a particular direction between repeated tests, was assessed with a 2-tailed paired samples ttest $(p \leq 0.05)$.

Relative reliability was assessed by calculating the ICC $(2,1)$. Weir et al. [48] stated that for test-retest situations, the design dictates that trials are crossed with subjects and therefore lend themselves to analysis by 2-way models. These authors also stated that the 2-way model addressed both systematic and random error, therefore ICC equation 2,1 should be used. An ICC above 0.90 was considered very high, between 0.70 and 0.89 as high,

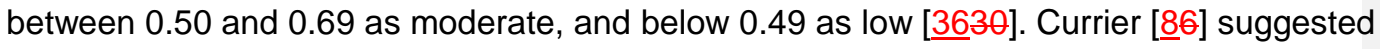
that an ICC value $>0.80$ was acceptable for clinical work. From the ICC value the Standard Error of Measurement (SEM) was calculated, which represented absolute reliability. It has been argued that the 2-way model ICC should be used when calculating the SEM as the 
systematic and random errors are considered separately $[24,48]$. The SEM was calculated using the following formula:

$\mathrm{SEM}=\mathrm{SD} \sqrt{ } 1-\mathrm{ICC}$

Where SEM = standard error of measurement, SD = the standard deviation of the sample, and ICC $=$ the calculated intraclass correlation coefficient.

\section{Results}

\section{Peak torque}

The three-way mixed factorial ANOVA showed no significant differences for the three fatigue indicators (fatigue index, percent decrease in performance and the slope of the regression equation) between the first week and second week of testing. There were also no significant differences between the two groups tested (healthy and healthy with a history of FAl), or the four speeds tested $\left(60,90,120\right.$ and $\left.180^{\circ} \cdot \mathrm{s}^{-1}\right)$ when observing all peak torque data. When studying the relative reliability results for the healthy subject's peak torque (table 1) the values ranged from moderate to very high depending on which fatigue determination method was used. The fatigue index showed moderate to high relative reliability, with speeds 60,90 , 120 and $180^{\circ} \cdot \mathrm{s}^{-1}$ showing ICC results of $0.60,0.74,0.88$ and 0.78 , respectively. Absolute reliability for the fatigue index showed SEM values of $0.94 \%, 1.14 \%, 1.03 \%$ and $1.36 \%$, for speeds $60,90,120$ and $180^{\circ} \cdot \mathrm{s}^{-1}$, respectively. The percent decrease in performance method showed high relative reliability, with speeds $60,90,120$ and $180^{\circ} \cdot \mathrm{s}^{-1}$ showing ICC results of $0.78,0.85,0.88$ and 0.77 , respectively. Absolute reliability for the percent decrease 
in performance showed SEM values of $0.93 \%, 0.64 \%, 0.52 \%$ and $0.94 \%$, for speeds 60,90 , 120 and $180^{\circ} \cdot \mathrm{s}^{-1}$, respectively. The slope of the regression equation showed high to very high relative reliability, with speeds $60,90,120$ and $180^{\circ} \cdot \mathrm{s}^{-1}$ showing ICC results of 0.85 , $\underline{0.91,0.93}$ and 0.91 , respectively. Absolute reliability for the slope of the regression equation showed SEM values of $0.06 \%, 0.08 \%, 0.02 \%$ and $0.07 \%$ for speeds $60,90,120$ and $180^{\circ}$. $\mathrm{s}^{-1}$, respectively. The slope of the regression equation was the most reliable method of fatigue determination, and the most reliable testing speed was $120^{\circ} \cdot \mathrm{s}^{-1}$.

When studying the relative reliability results for the healthy subjects with a history of FAI the peak torque values (table 1) ranged from moderate to very high depending on which fatigue indicator was used. The fatigue index showed high relative reliability, with speeds 60, 90, 120 and $180^{\circ} \cdot \mathrm{s}^{-1}$ showing ICC results of $0.79,0.77,0.71$ and 0.75 , respectively. Absolute reliability for the fatigue index showed SEM values of $0.41 \%, 0.41 \%, 0.44 \%$ and $0.77 \%$ for speeds $60,90,120$ and $180^{\circ} \cdot \mathrm{s}^{-1}$, respectively. The percent decrease in performance method showed moderate to very high relative reliability, with speeds $60,90,120$ and $180^{\circ}$. $\mathrm{s}^{-1}$ showing ICC results of $0.69,0.71,0.93$ and 0.80 , respectively. Absolute reliability for the percent decrease in performance showed SEM values of $0.40 \%, 0.35 \%, 0.26 \%$ and $0.82 \%$ for speeds $60,90,120$ and $180^{\circ} \cdot \mathrm{s}^{-1}$, respectively. The slope of the regression equation showed high to very high reliability, with speeds $60,90,120$ and $180^{\circ} \cdot \mathrm{s}^{-1}$ showing ICC results of $0.91,0.80,0.92$ and 0.83 , respectively. Absolute reliability for the slope of the regression equation showed SEM values of $0.04 \%, 0.07 \%, 0.01 \%$ and $0.03 \%$ for speeds 60 ,

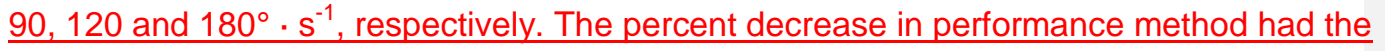
highest reliability $(\mathrm{ICC}=0.93, \mathrm{SEM}=0.26)$ when testing at $120^{\circ} \cdot \mathrm{s}^{-1}$. This was followed by the slope of the regression equation $(\mathrm{ICC}=0.92, \mathrm{SEM}=0.01)$ when testing at $120^{\circ} \cdot \mathrm{s}^{-1}$. 
$\underline{\text { Total work }}$

The three-way mixed factorial ANOVA showed no significant differences for the three fatigue indicators (fatigue index, percent decrease in performance and the slope of the regression equation) between the first week and second week of testing. There were also no significant differences between the two groups tested (healthy and healthy with a history of FAI), or the four speeds tested $\left(60,90,120\right.$ and $\left.180^{\circ} \cdot \mathrm{s}^{-1}\right)$ when observing all total work data. When studying the relative reliability results for the healthy subject's total work (table 2) the values ranged from moderate to very high depending on which fatigue determination method was used. The fatigue index showed moderate to high reliability, with speeds $60,90,120$ and $180^{\circ} \cdot \mathrm{s}^{-1}$ showing ICC results of $0.65,0.78,0.64$ and 0.60 , respectively. Absolute reliability for the fatigue index showed SEM values of $1.26 \%, 0.86 \%, 1.17 \%$ and $1.08 \%$ for speeds $60,90,120$ and $180^{\circ} \cdot \mathrm{s}^{-1}$, respectively. The percent decrease in performance method showed high relative reliability, with speeds $60,90,120$ and $180^{\circ} \cdot \mathrm{s}^{-1}$ showing ICC results of $0.82,0.81,0.81$ and 0.82 , respectively. Absolute reliability for the percent decrease in performance showed SEM values of $0.99 \%, 0.91 \%, 0.89 \%$ and $0.82 \%$ for speeds $60,90,120$ and $180^{\circ} \cdot \mathrm{s}^{-1}$, respectively. The slope of the regression equation showed high to very high relative reliability, with speeds $60,90,120$ and $180^{\circ} \cdot \mathrm{s}^{-1}$ showing ICC results of $0.87,0.86,0.92$ and 0.92 , respectively. Absolute reliability for the slope of the regression equation showed SEM values of $0.04 \%, 0.05 \%, 0.02 \%$ and $0.03 \%$ for speeds $60,90,120$ and $180^{\circ} \cdot \mathrm{s}^{-1}$, respectively. The results showed the slope of the regression to be the most reliable method of fatigue determination, when testing at a speed of $120^{\circ}$. 
When studying the reliability results for the subjects with a history of FAI the total work values (table 2) ranged from moderate to very high depending on which fatigue determination method was used. The fatigue index showed moderate to high reliability, with speeds $60,90,120$ and $180^{\circ} \cdot \mathrm{s}^{-1}$ showing ICC results of $0.74,0.71,0.81$ and 0.62 , respectively. Absolute reliability for the fatigue index showed SEM values of $0.85 \%, 0.92 \%$, $\underline{0.58 \%}$ and $0.61 \%$ for speeds $60,90,120$ and $180^{\circ} \cdot \mathrm{s}^{-1}$, respectively. The percent decrease in performance method showed moderate to high relative reliability, with speeds $60,90,120$ and $180^{\circ} \cdot \mathrm{s}^{-1}$ showing ICC results of $0.78,0.75,0.80$ and 0.60 , respectively. Absolute reliability for the percent decrease in performance showed SEM values of $0.19 \%, 0.25 \%$, $0.26 \%$ and $0.73 \%$ for speeds $60,90,120$ and $180^{\circ} \cdot \mathrm{s}^{-1}$, respectively. The slope of the regression equation showed low to very high relative reliability, with speeds $60,90,120$ and $180^{\circ} \cdot \mathrm{s}^{-1}$ showing ICC results of $0.86,0.84,0.90$ and 0.71 , respectively. Absolute reliability for the slope of the regression equation showed SEM values of $0.02 \%, 0.03 \%, 0.01 \%$ and

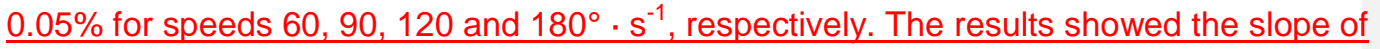
the regression to be the most reliable method when testing at $120^{\circ} \cdot \mathrm{s}^{-1}$.

Number of repetitions to fatigue

A three-way mixed factorial ANOVA showed no significant differences for the number of repetitions to fatigue between the first week and second week of testing. There were also no significant differences between the two groups tested (healthy and healthy with a history of FAI), or the four speeds tested $\left(60,90,120\right.$ and $\left.180^{\circ} \cdot \mathrm{s}^{-1}\right)$. The number of repetitions to fatigue was correlated to the ICC reliability values to see if a relationship was present. The healthy subjects produced $r$ values of 0.16 and 0.02 , for testing session 1 and testing session 2 , respectively. The healthy subjects with a history of FAl produced r values of 0.41 
and 0.17 , for testing session 1 and testing session 2 , respectively. The results showed there was no relationship present when correlating the number of repetitions to fatigue with the $\underline{\text { ICC } \text { reliability value. }}$

\begin{abstract}
Peak torque
All peak torque data found no significant differences $(p \leq 0.05)$ between test 1 and test 2 for any of the fatigue indicators or speeds tested. When observing the reliability results for the healthy subject's peak torque (table 1) the values ranged from moderate to very high depending on which fatigue determination method was used. The fatigue index showed moderate to high reliability. The speeds $60,90,120$ and $180^{\circ} \cdot s^{-1}$ showed reliability results (ICC \pm SEM) of $0.60 \pm 0.94,0.74 \pm 1.14,0.88 \pm 1.03$ and $0.78 \pm 1.36$ respectively. The percentage decrease in performance method showed high reliability. The speeds $60,90,120$ and $180^{\circ}$. $s^{-1}$ showed reliability results of $0.78 \pm 0.93,0.85 \pm 0.64,0.88 \pm 0.52$ and $0.77 \pm 0.94$ respectively. The slope of the regression equation showed high to very high reliability. The speeds 60,90 , 120 and $180^{\circ} \cdot \mathrm{s}^{-1}$ showed reliability results of $0.85 \pm 0.06,0.91 \pm 0.08,0.93 \pm 0.02$ and $0.91 \pm 0.07$ respectively. The slope of the regression was the most reliable method of fatigue determination, and the most reliable testing speed was $120^{\circ} \cdot \mathrm{s}^{-1}$.
\end{abstract}

When studying the reliability results for the injured subject's peak torque (table 2) the values ranged from moderate to very high depending on which fatigue indicator was used. The fatigue index showed high reliability. The speeds $60,90,120$ and $180^{\circ} \cdot \mathrm{s}^{-1}$-showed reliability results (ICG 1 SEM) of $0.79 \pm 0.41,0.77 \pm 0.41,0.71 \pm 0.44$ and $0.75 \pm 0.77$ respectively. The percentage decrease in performance method showed moderate to very high reliability. The speeds $60,90,120$ and $180^{\circ} \cdot \mathrm{s}^{-1}$-showed reliability results of $0.69 \pm 0.40,0.71 \pm 0.35$, 
$0.93 \pm 0.26$ and $0.80 \pm 0.82$ respectively. The slope of the regression equation showed high to very high reliability. The speeds $60,90,120$ and $180^{\circ} \cdot \mathrm{s}^{-1}$ showed reliability results of $0.91 \pm 0.04,0.80 \pm 0.07,0.92 \pm 0.01$ and $0.83 \pm 0.03$ respectively. The percentage decrease in performance method had the highest reliability $(0.93 \pm 0.26)$ when testing at $120^{\circ} \cdot \mathrm{s}^{-1}$. This was followed by the slope of the regression equation which showed reliability values of $0.92 \pm 0.01$ when testing at $120^{\circ} \cdot s^{-1}=$

\section{Total work}

All total work data found no significant differences $(p \leq 0.05)$ between test 1 and test 2 for any of the fatigue indicators or speeds tested. When observing the reliability results for the healthy subject's total work (table 3 ) the values ranged from moderate to very high depending on which fatigue determination method was used. The fatigue index showed moderate to high reliability. The speeds $60,90,120$ and $180^{\circ}, s^{-1}$ showed reliability results (ICC \pm SEM) of $0.65 \pm 1.26,0.78 \pm 0.86,0.64 \pm 1.17$ and $0.60 \pm 1.08$ respectively. The percentage decrease in performance method showed high reliability. The speeds $60,90,120$ and $180^{\circ}$ $s^{-1}$ showed reliability results of $0.82 \pm 0.99,0.81 \pm 0.91,0.81 \pm 0.89$ and $0.82 \pm 0.82$ respectively. The slope of the regression equation showed high to very high reliability. The speeds 60,90 , 120 and $180^{\circ} \cdot \mathrm{s}^{-1}$ showed reliability results of $0.87 \pm 0.04,0.86 \pm 0.05,0.92 \pm 0.02$ and $0.92 \pm 0.03$ respectively. The results showed the slope of the regression to be the most reliable method of fatigue determination, when testing at a speed of $120^{\circ}$.

When studying the reliability results for the injured subject's total work (table 4) the values fanged from low to very high depending on which fatigue determination method was used. 


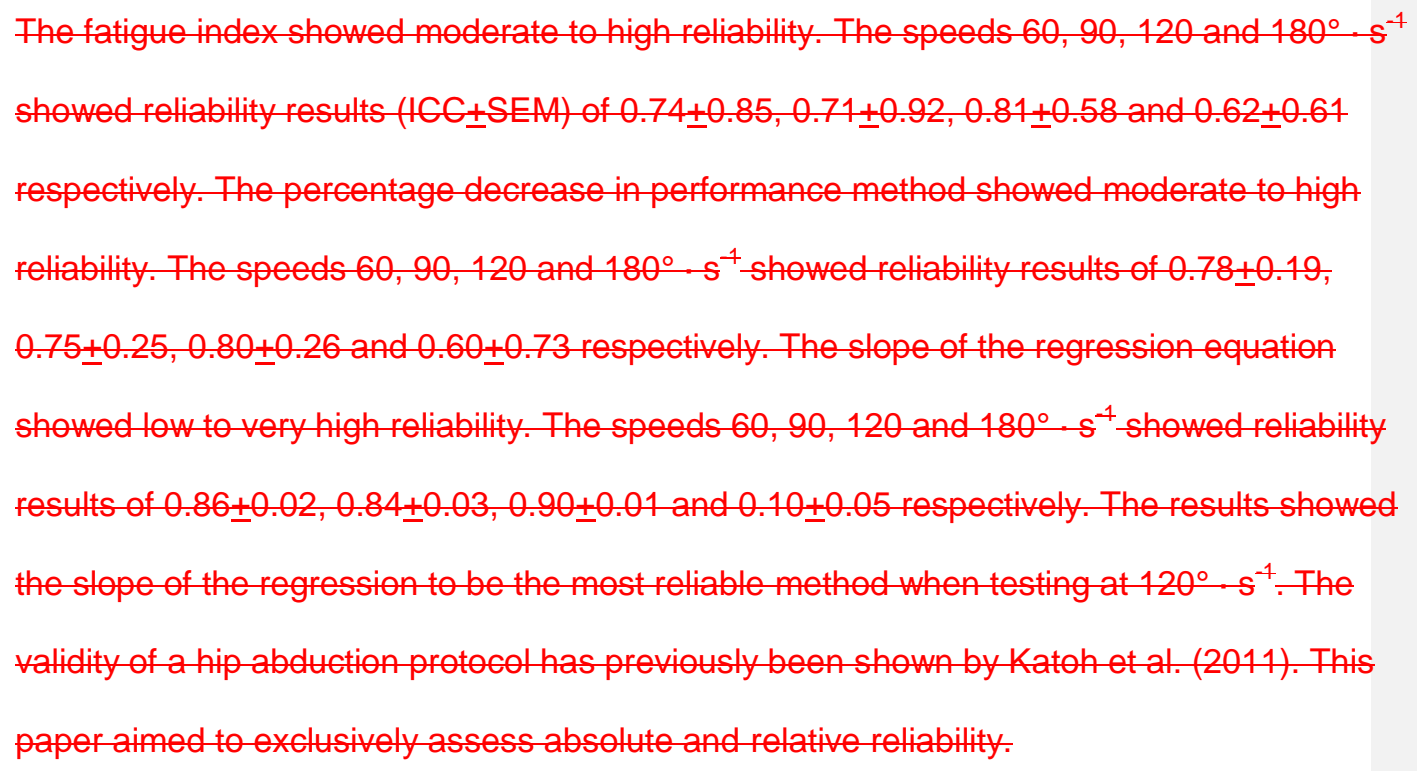

\section{Discussion}

The aim of this study was to examine the test-retest reliability of fatigue measures calculated from peak torque and total work during isokinetic speeds of $60,90,120$ and $180^{\circ} \cdot \mathrm{s}^{-1}$ during an isokinetic hip abductor-adductor fatigue protocol. The main findings that emerged from the study were firstly, the slope of the regression equation was the most reliable method of fatigue determination in healthy subjects and healthy subjects with a history of FAI injured subjects, when using peak torque or total work values, and secondly, the most reliable fatigue measures occurred at the speed of $120^{\circ} \cdot \mathrm{s}^{-1}$.

The choice of either peak torque or total work to assess average performance during a fatigue test does not seem to influence relative or absolute reliability. The same conclusion applies to the speed of the isokinetic dynamometer as relative and absolute 
reliability values were not influenced by a change in speed. When observing the peak torque values at $60,90,120$ and $180^{\circ} \cdot \mathrm{s}^{-1}$ the relative reliability values (ICC) for the slope of the line measure were consistently between $0.85-0.93$ for the healthy subjects and 0.80-0.92 for the healthy subjects with a history of FAI injured subjects. Absolute reliability (SEM) also produced consistently low values between $0.02 \%-0.08 \%$ for the healthy subjects, and $0.01 \%-0.07 \%$ for the healthy subjects with a history of FAI injured subjects. The same can be observed with the total work values for the different isokinetic dynamometry speeds, as relative reliability for the slope of the line measure were between $0.86-0.92$ for healthy subjects, and $0.84-0.90$ for the healthy subjects with a history of FAI injured subjects, with the exception of $180^{\circ} \cdot s^{-1}$ which unexpectedly produced a very low ICC value of 0.10 . Absolute reliability values were again consistent with the total work measure producing values between $0.02 \%-0.05 \%$ for the healthy subjects and $0.01 \%-0.05 \%$ for the healthy subjects with a history of FAI injured subjects. It is also apparent from the above results that the type of subjects tested (healthy or healthy with a history of FAI) did not influence relative of absolute reliability results.

The different fatigue determination methods did produce large variations in relative and absolute reliability values. The slope of the line measurement consistently produced high relative and absolute reliability values, , with the exception of total work at $180^{\circ} \cdot \mathrm{s}^{-1}$ in the injured subjects. Whereas, the fatigue index and the percentage decrease in performance produced lower and more variable relative and absolute reliability values (tables 1-2 1-4). 
A limited number of studies have looked at the reliability of different fatigue measures. Bosquet et al. [3] found high relative reliability (peak torque ICC's $=0.82-0.88$, total work ICC's $=0.81-0.87)$ for the slope of the line. Pincivero et al. [3933] studied the reliability of the fatigue index and the slope of the line during isokinetic quadriceps femoris muscle fatigue. The authors found moderate to high ICC's for the non-dominant leg (0.78-0.92) and high ICC's for the dominant leg $(0.82-0.89)$ when analysed by the slope. These results agree with the present study as we found the slope of the line to be the most reliable method when observing both when looking at both relative and absolute reliability.

The appropriateness of a method to objectively quantify muscle strength or endurance is dependent upon its reliability and the inherent error associated with that method. Piva et al. [4034] illustrated high test-retest reliability of an isometric hip abduction protocol (ICC $=0.92$ ). Claiborne et al. [ㅁu] showed that peak torque measurements during isokinetic hip abduction at $60^{\circ} \cdot \mathrm{s}^{-1}$ displayed high test-retest reliability (ICC range $=0.81-0.91$ ) Although not directly related to the hip, Feiring et al. [1210] showed that quadriceps peak torque and total work displayed high test-retest reliability $(\mathrm{ICC}=0.96$ and ICC $=0.97$, respectively). It should be recognised that the ability of reproducing the testing protocol with respect to adequate calibration, gravity correction, and standard patient set up in the current study was likely to have improved accuracy, and should be deemed important components for improving the reliability of a test $[21,38,39,4918,32,33,39]$.

The accuracy to which these protocols are reproducible is also a critical factor as determined by the standard error of measurement (SEM). Although high reliability coefficients (such as ICC's) have been previously reported for isokinetic strength, SEM 
values have received little attention in the literature. The SEM value in this study was expressed as a percentage in order to allow clinical usage of these measures. As demonstrated by the results of the current study, re-test values for peak torque and total work varied by $0.01-1.52 \%$ to the initial test. It should therefore, seem appropriate in future studies to attribute differences in isokinetic results to intervention, training improvements or injury, should they exceed the SEM values outlined in tables 1 and 2.1 to 4 .

There seems to be a lack of consensus in the literature on the most appropriate or reliable speed to be used for isokinetic dynamometry. The knee joint has been well documented with the majority of authors opting for a speed of $180^{\circ} \cdot \mathrm{s}^{-1}$. The hip, however, has very rarely been studied. The sparse literature that has tested the hip joint have either used the isokinetic dynamometer in its isometric mode [4034], or have tested at speeds of 60 and $90^{\circ} \cdot \mathrm{s}^{-1}$ [4236]. Ferber et al. [1311] found that during running at $3.65 \mathrm{~m} / \mathrm{s}(13.2 \mathrm{~km} / \mathrm{hr})$ over a $25 \mathrm{~m}$ distance, the peak angular velocity for hip flexion was $103.5^{\circ} \cdot \mathrm{s}^{-1}$. The present study found $120^{\circ} \cdot \mathrm{s}^{-1}$ to be the most reliable testing speed, so even though this speed may be far from 'explosive sporting movement' velocities, it may replicate speeds from more endurance based activities as shown by Ferber et al. [1311].

Both peak torque and total work decreased during the test. Three methods were used to quantify this force reduction: the fatigue index [2838], percentage decrease in performance [1815] and the slope [3933]. The fatigue index and the percent decrease in performance measure the percentage of force reduction throughout the test. The slope 
represents the rate of decrease in performance. The main assumption, stated by Bosquet et al. [3], for using this measure is the linearity of the relationship between peak torque or total work and the number of repetitions.

Previous studies have reported a linear relationship between performance and the number of repetitions during 20 [를] and 30 [4638] maximal reciprocal concentric contractions. The slope could therefore be used to quantify muscle fatigue. However, Bosquet et al. [3] stated that there was a tendency of the line to plateau after 40 repetitions, and suggests that an exponential model would be more appropriate than a linear one to fit performance data measured for longer protocols. Studies by Gerdle and Elert [1714] and Larsson et al. [3026] also agree with the finding of Bosquet et al. [3]. However, the present study did not use a fixed number of repetitions and one subject reached 67 repetitions before 3 contractions were below $50 \%$ of their maximum peak torque. This subject still presented with a linear model, rather than an exponential decrease which would contrast with the above literature. The above studies were all performed on the knee, whereas the present study was fatiguing the hip musculature. We would recommend that future investigators examine and plot their data before choosing the slope of the line as their fatigue determination method, as a linear model is required. As a point of interest the number of repetitions to fatigue was correlated to the ICC reliability values to see if a relationship was present. However, the results showed no correlation between these two variables.

Limited research has focused on fatigue protocols of the hip musculature $[6,424,36]$. Current theory suggests that the hip abductors play a critical role in controlling foot 
placement during ambulation. A deficit at the hip abductors may alter foot placement, causing the foot to contact the ground in a more adducted position [1512]. In patients with FAI this potentially increases the chance of rolling over on the ankle, inducing a lateral ankle sprain. It has been suggested that fatigue of the hip abductors may also cause these deficits at the ankle joint [1512]. Therefore, it was crucial to develop a reliable hip fatiguing protocol for the hip abductors, so that research can continue to investigate this phenomenon.

\section{$\underline{\text { The results from the current study showed that the isokinetic dynamometer was a reliable }}$} device for testing the fatigability of the hip abductors in both healthy individuals but also healthy individuals with a history of FAI. Many individuals in the sporting population suffer from a history of FAI, and the results from this study conclude that clinicians and other health professionals can perform isokinetic testing protocols on the hip abductors with confidence that the protocol is reliable, in not only healthy individuals, but also the large population of healthy individuals with a history of FAI.

In summary, the most reliable fatigue determination method for the hip abductors was the slope of the regression equation, when testing at a speed of $120^{\circ} \cdot \mathrm{s}^{-1}$. However, it is recommended that future investigators examine and plot their data before choosing this as their fatigue indicator, as a linear model is required. The choice of either peak torque or total work to assess performance during a fatigue test did not influence relative or absolute reliability. The between-day reliability that was performed in the present study has valuable research and clinical relevance. Many athletic or rehabilitation activities typically involve multiple bouts of testing, sometimes with high-intensity muscle 
contractions. Protocols and methods used for testing should always be determined as reliable before testing commences, so that any differences that are reported can be reported as true. Clinicians can now perform an isokinetic fatigue protocol on the hip abductors with the reassurance that the procedure is reliable in both healthy individuals, and healthy individuals with a history of FAI.

\section{References}

1 Bahr R, Pena F, Shine J, Lew WD, Lindquist C, Tyrdal S, Engebretsen L. Mechanics of the anterior drawer and talar tilt tests. Acta Orthop Scand 1997; 68: 435-441

2 Bigland-Richie B, Woods JJ. Changes in muscular contractile properties and neural control during human muscular fatigue. Muscle Nerve 1984; 7: 691-699

3 Bosquet L, Maquet D, Forthomme B, Nowak N, Lehance C, Crosier JL. Effect of the lengthening of the protocol on the reliability of muscle fatigue indicators. Int $J$ Sports Med 2010; 31: 82-88

4 Brown LE, Whitehurst M, Bryant JR, Buchalter DN. Reliability of the Biodex System 2

Formatted: Font: Italic isokinetic dynamometer concentric mode. Isokinet Exerc Sci 1993; 3: 160-163

54 Brown LE, Whitehurst M, Gilbert R, Buchalter DN. The effect of velocity and gender Formatted: Indent: Left: $0 \mathrm{~cm}$, First on load range during knee extension and flexion exercise on an isokinetic device. $\mathrm{J}$

Orthop Sports Phys Ther 1995; 21: 107-112

664 Claiborne TL, Timmons MK, Pincivero DM. Test-retest reliability of cardinal plane isokinetic hip torque and EMG. J Electromyogr Kinesiol 2009; 19: 345-352 
| 75 Croisier JL, Ganteaume S, Binet J, Genty M, Ferret J. Strength imbalances and prevention of hamstring injury in professional soccer players. Am J Sports Med 2008;

36: $1469-1475$

| 66 Currier D. Elements of research in physical therapy. Baltimore: Williams and Wilkins; 1990

97 Docherty CL, Rybak-Webb K. Reliability of the anterior drawer and talar tilt tests using the LigMaster joint arthrometer. J Sport Rehabil 2009; 18: 389-397

108 Drouin JM, Valovich-McLeod TC, Shultz SJ, Gansneder BM, Perrin DH. Reliability and validity of the Biodex system 3 pro isokinetic dynamometer velocity, torque and position measurements. Eur J Appl Physiol 2004; 91: 22-29

119 Emery CA, Maitland ME, Meeuwisse WH. Test-retest reliability of isokinetic hip adductor and flexor muscle strength. Clin J Sport Med 1999; 9: 79-85

1210 Feiring DC, Ellenbecker TS, Dershield GL. Test-retest reliability of the Biodex isokinetic dynamometer. J Orthop Sport Phys Ther 1990; 11: 298-300

1311 Ferber R, McClay-Davis I, Williams DS. Gender differences in lower extremity mechanics during running. Clin Biomech 2003; 18: 350-357

14 Forestier $N$, Toschi $P$. The effects of an ankle destabilization device on muscular activity while walking. Int J Sports Med 2005; 26: 464-470

1512 Friel K, McLean N, Myers C, Caceres M. Ipsilateral hip abductor weakness after inversion ankle sprain. J Athl Train 2006; 41: 74-78.

1613 Gear WS. Effect of different levels of localized muscle fatigue o knee position sense. Sports Med 2011; 10: 725-730 
1714 Gerdle B, Elert J. The temporal occurrence of the mean power frequency shift of the electromyogram during maximal prolonged dynamic and static working cycles. Int J Sports Med 1994; 15 (Suppl 1): S32-S37

1815 Glaister M, Stone MH, Stewart AM, Hugues M, Moir GL. The reliability and validity of fatigue measures during short duration maximal intensity intermittent exercise. $J$ Strength Cond Res 2004; 18: 459-462

1916 Gleeson NP, Mercer TH. Reproducibility of isokinetic leg strength and endurance characteristics of adult men and woman. Eur J Appl Physiol 1992; 65: 221-228 2017 Gleeson NP, Mercer TH. The utility of isokinetic dynamometry in the assessment of human muscle function. Sports Med 1996; 21: 18-34

2118 Gross MT, Huffman GM, Phillips CN, Wray JA. Intramachine and intermachine reliability of the Biodex and the Cybex II for knee flexion and extension peak torque and angular work. J Orthop Sport Phys Ther; 1991; 13: 329-335

22 Harriss DJ, Atkinson G. Update - Ethical Standards in Sport and Exercise Science

Research. Int J Sports Med 2011; 32: 819-821

19 Harris D, Atkinson G. International Journal of Sports Medicine - Ethical Standards in Sport and Exercise Science Research. Int J Sports Med 2009; 30: 701-702

$\underline{23} 20$ Hislop HJ, Perrine JJ. The isokinetic concept of exercise. Phys Ther 1967; 47: $114-117$

24 Hopkins WG. Measures of reliability in sports medicine and science. Sports Med 2000; 30: 375-381

2521 Houweling TA, Head A, Hamzeh MA. Validity of isokinetic testing for previous hamstring injury detection in soccer players. Isokinet Exerc Sci 2009; 17: 213-220 
| 2622 Hubbard TJ, Kaminski TW. Kinaesthesia is not affected by functional ankle instability status. J Athl Train 2002; 37: 481-486.

2723 Janssen JC, Le-Ngoc L. Intratester reliability and validity of concentric measurements using a new hand-held dynamometer. Arch Phys Med Rehabil 2009;

90: $1541-1547$

2824 Kannus $P$. Isokinetic evaluation of muscular performance: implications for muscle testing and rehabilitation. Int J Sports Med 1994; 15 (Suppl 1): S11-S18

$\underline{29} 25$ Katoh M, Huragi Y, Uch ida M. Validity of isometric muscle strength

measurements of the lower limbs using a hand-held dynamometer and belt: a

comparision with an isokinetic dynamometer. J Phys Ther 2011; 23: 553-557

3026 Larsson B, Karlsson S, Eriksson M, Gerdle B. Test-retest reliability of EMG and

peak torque during repetitive maximum concentric knee extensions. J Electromyogr

Kinesiol 2003; 13: 281-287

31 Liu SH, Jason WJ. Lateral ankle sprains and instability problems. Clin Sports Med

1994; 13: 793-809

3227 Maffiuletti NA, Bizzini M, Desbrosses K, Babault N, Munzinger U. Reliability of

knee extension and flexion measurements using the Con-Trex isokinetic

dynamometer. Clin Physiol Funct Imaging 2007; 27: 346-353

3328 Makofsky H, Panicker S, Abbruzzese J, Aridas C, Camp M, Drakes J, Franco C,

Sileo $R$. Immediate effect of grade IV inferior hip joint mobilization on hip abductor

torque: a pilot study. J Manual Manipulative Ther 2007; 15: 103-111

3429 McNair PJ, Depledge J, Brettkelly M, Stanley SN. Verbal encouragement: effects on maximum effort voluntary muscle action. Br J Sports Med 1996; 30: 243-245 
35 Messina DF, Farney WC, DeLee JC. The incidence of injury in Texas high school

basketball: a prospective study among male and female athletes. Am J Sports

Med 1999; 27: 294-299

3630 Munro B. Statistical methods for health care research. Third edition. New York: Lippincott; 1997: 1-444

3731 Orri JC, Darden GF. Technica report: reliability and validity of the iSAM 9000 isokinetic dynamometer. J Strength Cond Res 2008; 22: 310-317

3832 Pincevero DM, Lephart SM, Karunakara. Reliability and precision of isokinetic strength and muscular endurance for the quadriceps and hamstrings. Int J Sports Med 1997; 18: 113-117

3933 Pincevero DM, Gear WS, Sterner RL. Assessment of the reliability of highintensity quadriceps femoris muscle fatigue. Med Sci Sports Exerc 2001; 33: 334338

4034 Piva SR, Teixeira P, Almeida G, Gil AB, DiGioia AM, Levison TJ, Fitzgerald GK.

Contribution of hip abductor strength to physical function in patients with total knee arthroplasty. Phys Ther 2011; 91: 225-233

4135 Reid, D. Assessment and rehabilitation of sports injury. New York: Churchill Livingstone, 1992.

$\underline{4236}$ Salavati M, Moghadam M, Ebrahimi I, Arab AM. Changes in postural stability with fatigue of lower extremity frontal and sagittal plane movers. Gait Posture 2007; 26: $214-218$

4337 Sole G, Hamren J, Milosavljevic S, Nicholson H, Sullivan SJ. Test-retest reliability of isokinetic knee extension and flexion. Arch Phys Med Rehabil 2007; 88: 626-631 


\section{Smith RW, Reischl SF. Treatment of ankle sprains in young athletes. Am J Sports}

Med 1986; 14: 465-471

45 Taylor NAS, Sanders RH, Howick IE, Stanley, SN. Static and dynamic assessment

of the Biodex dynamometer. Eur J Appl Physiol 1991; 62: 180-188

4638 Thorstensson A, Karlsson J. Fatiguability and fibre composition of human skeletal muscle. Acta Physiol Scand 1976; 98: 318-322

47 Weir JP, Evans SA, Housh ML. The effect of extraneous movements on peak torque and constant joint angle torque-velocity curves. J Orthop Sports Phys Ther 1996; 23: $\underline{302-308}$

48 Weir JP. Quantifying test-retest reliability using the intraclass correlation coefficient and the SEM. J Strength Cond Res 2005; 19: 231-240

4939 Winter DA, Wells RP, Orr GW. Error in the use of isokinetic dynamometers. Eur J Appl Physiol 1981; 46: 397-408

50 Wolfe MW, UhI TL, Mattacola CG, McCluskey LC. Management of ankle sprains. Am Fam Physician 2001; 63: 93-104

5140 Zawadzki J, Bober T, Siemienski A. Validity analysis of the Biodex system 3 dynamometer under static and isokinetic conditions. Acta of Bioeng Biomech 2010;

12: $25-32$

\section{List of Tables}

Table 1. Healthy subjects hip abductor peak torque

Table 2. Injured subjects hip abductor peak torque 
Table 3. Healthy subjects hip abductor total work
Table 4. Injured subjects hip abductor total work
Table 1. Muscle fatigue indicators from peak torque data during hip abduction.

Table 2. Muscle fatigue indicators from total work data during hip abduction.

\section{List of Figures}

Figure 1. Load rand as a percentage of the total test range of motion (ROM) during hip abduction. 